\title{
Neuroregulation of protein synthesis in odontoblasts of the first molar of the rat after wounding *
}

\author{
Daniel J. Chiego, Jr. ${ }^{1}$, James K. Avery ${ }^{1}$, and Robert M. Klein ${ }^{2}$ \\ ${ }^{1}$ Department of Oral Biology, University of Michigan, School of Dentistry, Ann Arbor, Michigan, USA; \\ ${ }^{2}$ Department of Anatomy/Division of Cell Biology, University of Kansas, College of Health Sciences and Hospital, \\ Kansas City, Kansas, USA
}

\begin{abstract}
Summary. Odontoblasts respond to occlusal trauma by increased elaboration of a matrix which is subsequently calcified to form reparative dentin. The purpose of the present study was to analyze quantitatively and compare the ability of odontoblasts to synthesize collagen after wounding in rats with an intact innervation (baseline) and in rats with sensory (inferior alveolar nerve, IAN) and/or sympathetic (superior cervical ganglion, $\mathrm{SCG}$ ) surgical denervation. Surgery was performed 7 days prior to wounding. All rats had $1 \mathrm{~mm}$ of enamel and dentin removed from the occlusal surface of the first mandibular molar (resected side) with the contralateral tooth serving as a control. Rats were killed $1 \mathrm{~h}$ after injection with ${ }^{3} \mathrm{H}$-proline on days $0,5,10$ or 15 after wounding, and mandibles were removed and processed for autoradiography. Grain counts were performed over odontoblasts throughout the pulp horns for each time period and for control and experimental molars in intact (baseline) and denervated groups. When compared to the control baseline, the experimental baseline data showed increased ${ }^{3} \mathrm{H}$-proline uptake throughout the study with a peak at 5 days. When compared to the baseline data, IAN and SCG results demonstrated a delay or attenuation of the protein synthetic response. The results indicate that the sensory and sympathetic neural components may regulate odontoblastic response to wounding.
\end{abstract}

Key words: Odontoblasts - Protein synthesis - Denervation - Neural regulation - Wound healing - Autoradiography - Rat

The primary response of odontoblasts to various stimuli, such as caries (Karjalainen and Soderling 1980, 1984), occlusal attrition (Sayegh 1967) and restorative procedures (Avery et al. 1974) is an increased production of protein (including collagen) that is secreted into the predentinal matrix and subsequently mineralized. This reparative dentinal response is an exaggeration of the incremental, appositional pattern of matrix deposition (Massler and Schour 1946), although in this case the response appears to be limited to the traumatized area (James and Schour 1950; Avery et al. 1974). How this response is initiated and

* Supported by a grant from the National Institutes of Health (DE05636)

Send offprint requests to: Dr. Daniel J. Chiego, Jr., Department of Oral Biology, University of Michigan, School of Dentistry, Ann Arbor, Michigan 48109, USA limited or whether the initial odontoblastic response is to cellular injury, bacterial toxins, or some other factor is not known.

Recently, several investigators have shown that autonomic and sensory nerves have an effect on the incorporation of tritiated precursors, proline and fucose, into newly forming alveolar bone (Chiego and Singh 1981) and predentin of the continuously erupting rat incisor (Chiego et al. 1983). These studies have shown that the loss of the afferent (sensory) or the visceral efferent (autonomic) or both of these neural components affects the metabolic activity of osteoblasts and/or odontoblasts. Whether the nerves mediate metabolic functions or are involved in inducing cells or tissues to differentiate is still equivocal.

The purpose of this study was to quantitatively evaluate discrete changes in the protein synthetic activity of odontoblasts by denervation of sensory and/or autonomic nerves known to be associated with odontoblasts and the dental pulp in conjunction with stimulation of reparative dentin formation.

\section{Materials and methods}

Eighty male Charles Rivers CD rats (150-160 g) were maintained on a $12 \mathrm{~h}$ light-dark cycle (lights on $06: 00-18: 00 \mathrm{~h}$ ) and separated into four groups of 20 rats: 1 baseline (intact innervation) and 3 resection groups. All rats had $1 \mathrm{~mm}$ of enamel and dentin removed from the occlusal surface of the right first mandibular molar by use of a \# 557 carbide crosscut fissue bur and an ultraspeed rotary handpiece cooled with an air-water spray on the experimental side. No occlusal reduction was performed on the contralateral (left) first molar which was used as a control. The baseline group had an intact innervation with wounding of the first molar. In the remaining three groups the inferior alveolar nerve (IAN), the superior cervical ganglion (SCG) or both (IAN + SCG) were resected unilaterally on the right side with the left first molar remaining as the control. Denervation was performed 7 days prior to wounding and 5 rats were killed on days $0,5,10$ or 15 in each treatment group. Rats were injected with $1 \mu \mathrm{Ci} / \mathrm{g}$. b. wt. ${ }^{3} \mathrm{H}$-proline (specific activity $=1.0 \mathrm{Ci} / \mathrm{mmole}$, New England Nuclear Co., Boston, MA) $1 \mathrm{~h}$ prior to killing at the appropriate time interval. Both surgery and euthanasia of the rats were performed between 10:00 and 12:00. The mandibles were removed and placed in $10 \%$ phosphate buffered formalin overnight, scraped free of soft tissue and decalcified in EDTA. The mandibles were subsequently dehydrated in a graded series 


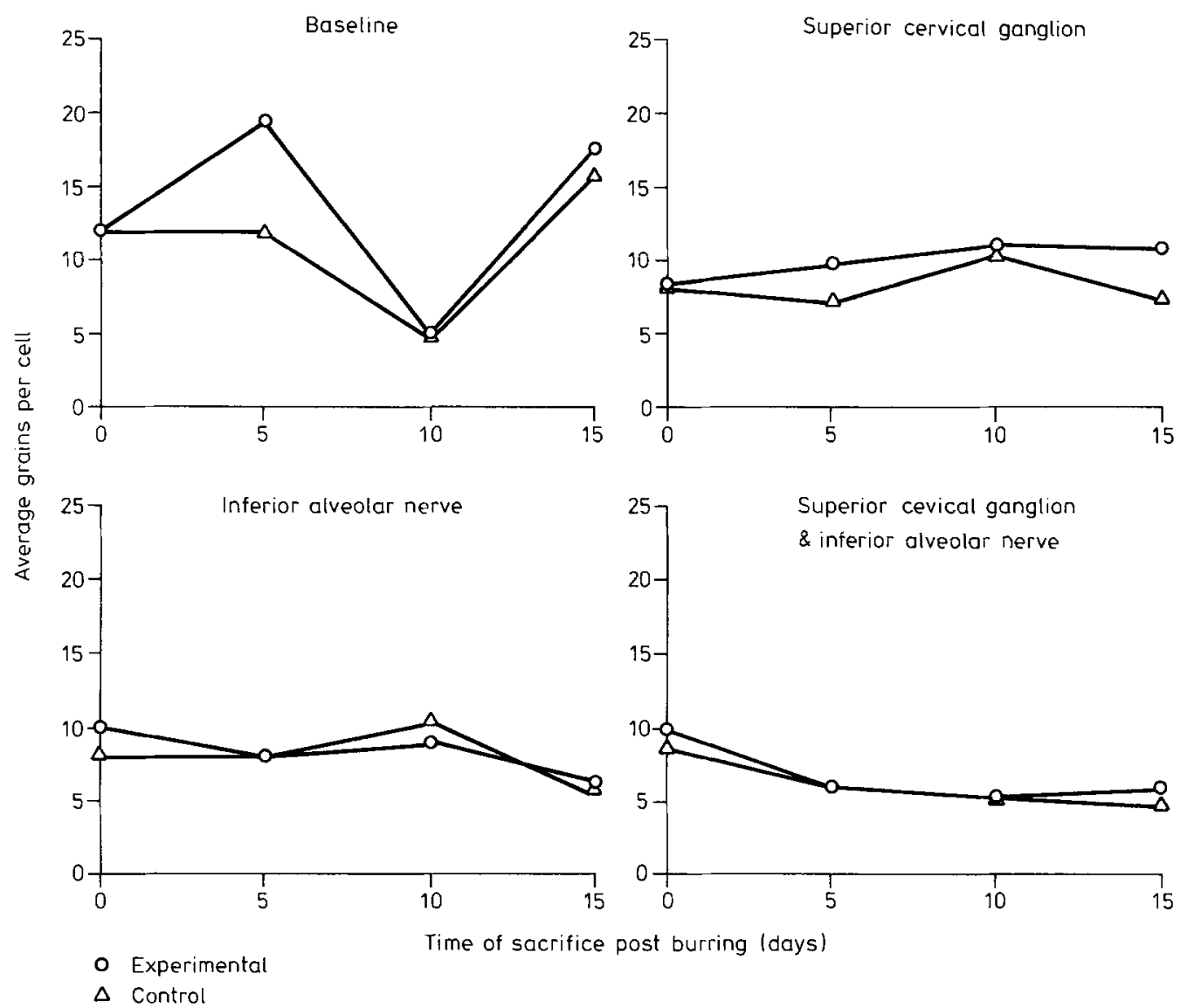

Fig. 1. Summary of grain-count analysis following ${ }^{3} \mathrm{H}$-proline injection into denervated and intact rats. Comparisons were statistically significant as indicated in Table 1. Baseline data refer to condition of intact innervation during tooth wounding and repair

of alcohols, embedded in paraffin, sectioned at $5 \mu \mathrm{m}$ and placed on glass slides. The slides were then dipped in Kodak NTB-2 emulsion and exposed at $4^{\circ} \mathrm{C}$ for 14 days. After developing and fixing, the autoradiograms were stained with Harris' hematoxylin and eosin and coverslipped. Reduced silver halide grains were counted over the odontoblastic cell bodies and $10 \mu \mathrm{m}$ into the predentin throughout the pulp horns for each time period and for all experimental and control procedures. Five adjacent sections from the mid-sagittal plane were used for each animal in this study for grain count analysis. Differences in pulpal areas were less than $5 \%$ for each tooth as determined by means of a Zeiss Interactive Digital Analysis System. Grain counts for right and left molars were compared by use of an unpaired $t$-test. Only histological sections that contained little or no observable inflammatory response were included for grain count analysis.

\section{Results}

The results of this study are shown graphically in Fig. 1, and the results of statistical analysis are provided in Table 1. The baseline, wounded group showed a significant increase in autoradiographic grains at 5 days after wounding when compared to the controls. There was a bimodal distribution in the experimental odontoblasts with a peak at 15 days after surgery. This was in contrast to the experimental odontoblasts which showed the initial response on 0 day. The amount of ${ }^{3} \mathrm{H}$-proline incorporated by the odontoblasts in the baseline teeth, experimental and control, was the highest of all four groups, with the baseline experimental odontoblasts the higher of the two within this group.

The odontoblasts in the IAN resected group (Fig. 1, Table 1) demonstrated an initial peak in the incorporation of ${ }^{3} \mathrm{H}$-proline compared to the contralateral control. From the initial time point until the end of this study at 15 days after wounding, the odontoblasts in the experimental teeth incorporated a decreasing amount of the tritiated precursor. Although there was a slight increase in the amount of precursor incorporated at 10 days after wounding, this was not significantly different from the contralateral control values.

The odontoblasts in the group of rats that had the SCG extirpated demonstrated significant differences between the experimental and control sides at 5 and 15 days after wounding. The experimental SCG odontoblasts showed a gradual increase in the incorporation of tritiated proline from the beginning of this study at 0 day until the end at 15 days. The odontoblasts in the contralateral control had a bimodal distribution, similar to the IAN group, and demonstrated a small peak on 0 day and at 10 days after wounding. In contrast to the previous three groups of rats, the IAN and SCG denervated odontoblasts demonstrated significant differences between the experimental and control groups at 0,5 and 10 days after wounding (Fig. 1). Both experimental and control odontoblasts showed peak incorporation of the ${ }^{3} \mathrm{H}$-proline at the initial time point. From 0 day to the termination of this study, both the experimental and control odontoblasts demonstrated a decrease in the amount of precursor incorporated, although the experi- 
Table 1. Summary of statistical results

\begin{tabular}{|c|c|c|c|c|}
\hline \multirow[t]{2}{*}{ Days after surgery } & \multicolumn{4}{|l|}{${ }^{3} \mathrm{H}$-proline } \\
\hline & 0 & 5 & 10 & 15 \\
\hline $\begin{array}{l}\text { Baseline cont. } \\
\text { Baseline expt. }\end{array}$ & $\begin{array}{l}11.71 \pm 0.52 \\
11.85 \pm 0.26\end{array}$ & $\begin{array}{l}11.62 \pm 0.25^{*} \\
19.29 \pm 0.65\end{array}$ & $\begin{array}{l}4.47 \pm 0.17 \\
4.80 \pm 0.27\end{array}$ & $\begin{array}{l}15.66 \pm 0.31 \\
17.47 \pm 1.17\end{array}$ \\
\hline $\begin{array}{l}\text { IAN expt. } \\
\text { IAN cont. }\end{array}$ & $\begin{array}{l}8.08 \pm 0.37^{*} \\
9.93 \pm 0.35\end{array}$ & $\begin{array}{l}7.86 \pm 0.38 \\
7.82 \pm 0.61\end{array}$ & $\begin{array}{l}8.08 \pm 0.37 \\
9.93 \pm 0.35\end{array}$ & $\begin{array}{l}5.49 \pm 0.32 \\
5.99 \pm 0.71\end{array}$ \\
\hline $\begin{array}{l}\text { IAN \& SCG cont. } \\
\text { IAN \& SCG expt. }\end{array}$ & $\begin{array}{l}8.38 \pm 0.61 * \\
9.77 \pm 0.20\end{array}$ & $\begin{array}{l}6.03 \pm 0.25 * \\
5.76 \pm 0.22\end{array}$ & $\begin{array}{l}4.92 \pm 0.22 * \\
5.76 \pm 0.22\end{array}$ & $\begin{array}{l}4.62 \pm 0.52 \\
5.83 \pm 0.16\end{array}$ \\
\hline $\begin{array}{l}\text { SCG cont. } \\
\text { SCG expt. }\end{array}$ & $\begin{array}{l}7.97 \pm 0.83 \\
8.32 \pm 0.25\end{array}$ & $\begin{array}{l}7.12 \pm 0.09^{*} \\
9.67 \pm 0.18\end{array}$ & $\begin{array}{l}10.2 \pm 0.07 \\
10.91 \pm 0.88\end{array}$ & $\begin{array}{r}7.25 \pm 0.23^{*} \\
10.72 \pm 1.22\end{array}$ \\
\hline
\end{tabular}

${ }^{*} \mathrm{p} \leqq 0.05$

mental odontoblasts showed a slight, but non-significant, increase at 15 days after wounding. This group (IAN + SCG resection) demonstrated the smallest number of reduced silver halide grains per cell of all 4 groups.

\section{Discussion}

The response of the rat molar to wounding may be similar to the normal differentiation processes that occur during the morphogenetic development of the teeth and throughout life in the continuously erupting incisor. The steps involved in the differentiation of odontoblasts include cessation of the cell division cycle, polarization, and initiation of predentin secretion (Ruch 1985). Epithelial-mesenchymal interactions play a role in normal morphogenesis (Ruch 1985) and in the continuous growth of the incisor (Lehmann and Slavkin 1984). Developmentally, the innervation of the tooth has been hypothesized to play a role in tooth initiation (Kollar and Lumsden 1979), although recent data indicate that innervation may not play an essential role in tooth patterning and initiation (Lumsden and Buchanan 1986). The growth of fetal tooth germs in culture (Osman et al. 1979), in the anterior chamber of the eye (Lumsden 1979) and under serum-free conditions (Slavkin et al. 1982) has demonstrated that, as in other systems, such as the developing small intestine (Montgomery et al. 1981), there is an intrinsic program for differentiation. This program is followed even under conditions that deprive the organ of normal in situ growth factors, hormones, and neural influences. However, in other in vivo systems nerves appear to modulate various cellular responses both during development (Klein and McKenzie 1980) and during regenerative processes (Maden 1978; Mailman and Dresden 1979). The present study was designed to determine the potential role of neural influences in the response of odontoblasts to tooth wounding.

In the present experiments, when the sympathetic and/ or sensory nerve supply was interrupted, the response to wounding was delayed or attenuated at the initial time period and nonexistent at the 5 day period. Previous experiments have attempted to delineate the role of nerves in the regulation of growth in continuously erupting rodent incisors and in the odontoblastic response to tooth wounding. Resection of the IAN results in a complex pattern of altered ${ }^{3} \mathrm{H}$-thymidine labelling in the progenitive compartments of the mouse incisor between 15 and 60 days after denervation (Chiego et al. 1981). In this system, the pre- odontoblasts are the most stable population, although there is a significant decrease in preodontoblastic labelling index 60 days after surgery. In contrast, chemical sympathectomy has little effect on the progenitive compartments except for a reduced labelling index in the stratum intermedium and stellate reticulum (Klein et al. 1981). The response of the tooth pulp and odontoblasts to wounding also appears to be modulated by neural influences. Denervation results in a delay of the cells proliferative response to wounding with the most dramatic delay occurring in the IAN + SCG group (Chiego et al. 1986). This neural regulation of the cell proliferative response to wounding appears to be analogous to the neurotrophic role of innervation in amphibian limb regeneration where the total number of cells in the cell cycle and/or the rate at which the cells cycle is nerve-dependent (Maden 1978). While neurotrophic regulation of limb regeneration primarily involves modulation of cell proliferation, neurotrophic regulation of collagen and collagenase activity has been demonstrated in that experimental system.

The focus of the present study was the effect of denervation on the incorporation of ${ }^{3} \mathrm{H}$-proline into protein as a marker of odontoblastic activity following wounding. Protein synthesis (including collagen and non-collagenous protein) by odontoblasts has been analyzed under normal, pathological and repair conditions in both animal and human teeth. Radiolabelled proline is incorporated into precollagenous polypeptide chains (Leblond 1963; Reith 1968; Weinstock and Leblond 1974). Odontoblasts incorporate ${ }^{3} \mathrm{H}$-proline at a rapid rate (label is seen over the rough endoplasmic reticulum 2 min after intravenous injection) and labelled procollagen molecules are extruded into the extracellular space where procollagen is transformed into tropocollagen and subsequently polymerized to form the collagen fibrils of the predentinal matrix (Weinstock and Leblond 1974). Odontoblasts synthesize Type-I collagen (Cournil et al. 1979; Wright and Leblond 1980), as compared to pulpal cells which synthesize both collagens I and III (Cournil et al. 1979; Wright and Leblond 1980). Collagen metabolism and ${ }^{3} \mathrm{H}$-proline incorporation are normally higher in continuously erupting rat, mouse and rabbit incisors than in molars, but higher in molars than in other oral tissues (i.e., periodontium and gingivae) (Orlowski and Doyle 1976; Orlowski 1977; Shuttleworth et al. 1979). In the present baseline studies, there was a relatively high, steady incorporation of ${ }^{3} \mathrm{H}$-proline at the 4 intervals analyzed corroborating the previous data from microchemical and autoradiographic studies. 
The results of the present experimental studies demonstrate that with intact nerve supply, odontoblasts respond to wounding by incorporating increased amounts of ${ }^{3} \mathrm{H}$ proline which peaks between 0 and 5 days. Numerous studies have compared dentinal and pulpal collagen and the incorporation of radiolabelled precursors in sound and carious teeth. In early carious lesions of dentin, Karjalainen et al. (1979) reported increased protein synthesis in the odontoblast-predentin region as measured by biochemical assays. ${ }^{3} \mathrm{H}$ - or ${ }^{14} \mathrm{C}$-proline incorporation in early dentinal lesions shows a pattern of increased labelling just below the lesion in the predentin matrix and decreased labelling in the odontoblasts, with no change in the pulp (Karjalainen and Soderling 1980). In advanced lesions there is a decrease of collagen formation locally in the predentin with an increase in odontoblastic and pulpal proline incorporation (Karjalainen and Soderling 1980). ${ }^{3} \mathrm{H}$-proline has also been used to assess reparative dentin formation in rats (Sayegh 1967). In these studies radiolabelled-proline incorporation is minimal or absent in the traumatized areas immediately after occlusal reduction (4, 12 and $24 \mathrm{~h}$ after injury), but increases dramatically at later time points. Trauma following high-speed drilling produces similar results: a dramatic hypoactivity immediately after the injury in the area under the cavity preparation (Searls 1975). Areas adjacent and opposite the cavity preparation are also affected, but the reduction in ${ }^{3} \mathrm{H}$-proline incorporation is less dramatic. Unpublished data from our laboratory indicate that odontoblasts in the interradicular areas of the molar show a greater incorporation of ${ }^{3} \mathrm{H}$-proline than the pulpal horns after removal of $1 \mathrm{~mm}$ of enamel and dentin. These differences may reflect a similar regional variation in the response to wounding. However, it must be noted that in the present studies there was no apparent destruction of odontoblasts in contrast to the deliberate trauma produced in other reported studies.

An extensive adrenergic innervation has been demonstrated in both the incisors (Larsson and Linde 1971) and molars (Avery et al. 1980) of rodents. The innervation has been observed in close association to blood vessels, but is more extensive in the apical region of the rat incisor pulp (Larsson and Linde 1971). Although some adrenergic endings have been found in close proximity to the odontogenic zone in adult mouse molars, the majority of endings are vascular-related (Avery et al. 1980). Therefore, in the present studies, sympathetic denervation-induced changes in ${ }^{3} \mathrm{H}$-proline incorporation may be related to alterations in blood supply which indirectly affect dentinogenesis (Bishop and Dorman 1963).

In the case of IAN resection, indirect effects of sensory deprivation cannot be eliminated from consideration. Contralateral effects of denervation have been demonstrated with ${ }^{3} \mathrm{H}$-fucose (Chiego et al. 1983) and ${ }^{3} \mathrm{H}$-thymidine (Chiego et al. 1981) following IAN resection. Unilateral resection of the IAN probably results in altered chewing patterns. For example, rats in the present study could have been chewing more on the left side than on the right, resulting in secondary dentin formation in the control molar. This may explain the steady increase in ${ }^{3} \mathrm{H}$-proline incorporation in controls throughout the study.

In regard to direct effects of innervation and interaction of neural components in their regulation of tooth function, Weatherred (1965) demonstrated a transient decrease followed by an increase in intrapulpal pressure following IAN stimulation. A decrease in pressure following stimulation of the SCG was observed. While it is difficult to translate changes in pulpal pressure to metabolic alterations, there has been no evidence for a completely opposing interaction betwen the sympathetic and sensory innervation. In the case of cavity preparation and restoration IAN or IAN + SCG resection produces similar changes: a dramatic increase in dentin deposition both 14 and 35 days after cavity preparation (Avery et al. 1974). Resection of the superior cervical ganglion does not result in observable increases in dentin formation. Denervation has also been shown to affect the quality of the dentin produced. For example, dentinal changes after IAN resection include globular dentin with irregular calcification (Rehak 1963; Avery et al. 1971). In addition, autoradiographic studies demonstrate alterations in glycoprotein synthesis in the continuously erupting rodent incisor. In these studies IAN or SCG resection results in increased ${ }^{3} \mathrm{H}$-fucose incorporation into the predentinal matrix 2 weeks after surgery. The highest grain counts occur after combined (IAN + SCG) resection (Chiego et al. 1983). Percentage changes in ${ }^{3} \mathrm{H}$-fucose incorporation do not completely reflect these changes because of contralateral effects of the denervation procedure. In all cases in the present experiments in which either the IAN, SCG or IAN + SCG were resected, the odontoblasts showed a significantly lower amount of ${ }^{3} \mathrm{H}$-proline incorporated when compared to that in either the experimental or control baseline cells. Of these groups the IAN + SCG resection demonstrated the fewest grains. These data are indicative of a response similar to the apparent interaction between sensory and autonomic nerve components in relation to radiolabelled fucose incorporation in odontoblasts following denervation (Chiego et al. 1983) and in the proliferation of pulpal cells during repair responses (Chiego et al. 1986). Recently, studies reported by Kubota et al. (1985) in the continuously erupting rat incisor suggest that after trigeminal nerve resection there is a decrease in odontoblastic differentiation with no effect on the molars. In contrast, after resection of the superior cervical sympathetic nerves, the investigators found an increase in pulpal collagen in both the incisors and molars. In the case of double neurotomy the results were similar to those following sympathectomy. The data agree with our present study in which we found that extirpation of the superior cervical ganglion resulted in a significant increase in ${ }^{3} \mathrm{H}$-proline incorporation into odontoblasts on the experimental compared to the control side at 5 and 15 days following wounding. Although the interaction between different neural components is not clear, the data from the present study demonstrate that the autonomic and sensory innervation influence the protein-synthetic repair processes of odontoblasts following wounding of the teeth.

\section{References}

Avery JK, Strachan DS, Corpron RE, Cox CF (1971) Morphological studies of the altered pulps of the New Zealand white rabbit after resection of the inferior alveolar nerve and/or the superior cervical ganglion. Anat Rec 171:495-508

Avery JK, Cox CF, Corpron RE (1974) The effects of combined nerve resection and cavity preparation and restoration on response dentin formation in rabbit incisors. Arch Oral Biol 19:539-548

Avery JK, Cox CF, Chiego DJ Jr (1980) Presence and location of adrenergic nerve endings in the dental pulps of mouse molars. Anat Rec 198:59-71 
Bishop IG, Dorman HL (1963) Changes in perfusion pressure in the mandibular artery induced by functional alterations of the superior cervical ganglion. J Dent Res 42: 777-782

Chiego DJ Jr, Singh IJ (1981) Evaluation of the effects of sensory denervation on osteoblasts by ${ }^{3} \mathrm{H}$-proline autoradiography. Cell Tissue Res 217:569-576

Chiego DJ Jr, Klein RM, Avery JK (1981) Tritiated thymidine autoradiographic study of the effects of inferior alveolar nerve resection on the proliferative compartments of the mouse incisor formative tissues. Arch Oral Biol 26:83-89

Chiego DJ Jr, Fisher MA, Avery JK, Klein RM (1983) Effects of denervation of ${ }^{3} \mathrm{H}$-fucose incorporation by odontoblasts in the mouse incisor. Cell Tissue Res 230:197-203

Chiego DJ Jr, Klein RM, Avery JK, Gruhl IM (1986) Denervation induced changes in cell proliferation in the rat molar after wounding. Anat Rec $214: 348-352$

Cournil I, Leblond CP, Pomponio J, Hand AR, Sederlof A, Martin GR (1979) Immunohistochemical localization of procollagens. I. Light microscopic distribution of procollagen I, III and IV antigenicity in the rat incisor tooth by the indirect peroxidaseanti-peroxidase method. J Histochem Cytochem 27:1059-1069

James VE, Schour I (1955) Early dentinal and pulpal changes following cavity preparations and filling materials in dogs. Oral Surg 8:1305-1314

Karjalainen S, Soderling E (1980) The autoradiographic pattern of the in vitro uptake of proline by the coronal areas of intact and carious human teeth. Arch Oral Biol 24:909-915

Karjalainen S, Soderling E (1984) Dentino-pulpal collagen and the incorporation of ${ }^{3} \mathrm{H}$-proline by sound and carious human teeth in vitro. J Biol Buccale 12:309-316

Karjalainen S, Soderling E, Kuutti-Savolainen E-R (1979) Predentinal collagen and pulpal prolyl hyroxylase of intact and carious human teeth. Arch Oral Biol 24:555-559

Klein RM, McKenzie JC (1980) Pattern of crypt cell proliferation in the pre- and post-closure ileum of the neonatal rat: Effects of sympathectomy. Cell Tissue Res 206:387-394

Klein RM, Chiego DJ Jr, Avery JK (1981) Effects of chemical sympathectomy on cell proliferation in the progenitive compartments of the neonatal mouse incisor. Arch Oral Biol $26: 319-325$

Kollar EJ, Lumsden AGS (1979) Tooth morphogenesis: The role of the innervation during induction and pattern formation. $J$ Biol Buccale 7:49-60

Kubota K, Yonaga T, Hosaka K, Katayama T, Nagae K, Shibanai S, Sato Y, Takada K (1985) Experimental morphological studies on the functional role of pulpal nerves in dentinogenesis. Anat Anz (Jena) 158:323-336

Larsson P-A, Linde A (1971) Adrenergic vessel innervation in the rat incisor pulp. Scand J Dent Res 79:7-12

Leblond CP (1963) Elaboration of dentinal collagen in odontoblasts as shown by radioautography after injection of labelled glycine and proline. Ann Histochim $8: 43-50$

Lehmann R, Slavkin HC (1984) Identification of inner and outer cell proliferation centers during fetal tooth morphogenesis. J Craniofac Genet Dev Biol 4:47-57

Lumsden AGS (1979) Pattern formation in the molar dentition of the mouse. In: Tooth Morphogenesis and Differentiation (Edited by Ruch JV), Editions SPNMD, Paris, p 85

Lumsden AGS, Buchanan JAG (1986) An experimental study of timing and topography of early tooth development in the mouse embryo with an analysis of the role of innervation. Arch Oral Biol 31:301-311

Maden M (1978) Neurotrophic control of the cell cycle during amphibian limb regeneration. J Embryol Exp Morphol 48:169-175

Mailman ML, Dresden MH (1979) Denervation effects of newt limb regeneration: Collagen and collagenase. Dev Biol $71: 60-70$

Massler M, Schour I (1946) The appositional life span of the enamel and dentin-forming cells. I. Human deciduous teeth and first permanent molars. J Dent Res 25:145-150

Montgomery RK, Sybicki MA, Grand RJ (1981) Autonomous biochemical and morphological differentiation in fetal rat intestine transplanted at 17 and 20 days of gestation. Dev Biol 87:76-84

Orlowski WA (1977) The turnover of collagen in the dental pulp of rat incisors. J Dent Res 56:437-440

Orlowski WA, Doyle JL (1976) Collagen metabolism in the pulps of rat teeth. Arch Oral Biol 21:391-392

Osman A, Karcher-Djuricic V, Ruch JV (1979) Differenciation in vitro du materiel molaire presomptif de l'embryon de souris. C R Acad Sci Paris 289:149-151

Rehak JR (1963) Course and resection of the inferior alveolar nerve in the albino rat. J Dent Res 42:1159-1168

Reith EJ (1968) Ultrastructural aspects of dentinogenesis. In: Dentine and Pulp (Edited by Symons, NBB), Livingstone, Edinburgh, pp 19-41

Ruch JV (1985) Odontoblast differentiation and the formation of the odontoblast layer. J Dent Res 64:489-498

Sayegh F (1967) $\left[{ }^{3} \mathrm{H}\right]$-proline and tetracycline as marking agents in the study of reparative dentin formation. Oral Surg 23:221-229

Searls JC (1975) Radioautographic evaluation of changes induced in the rat incisor by high-speed cavity preparation. J Dent Res $54: 174-182$

Shuttleworth CA, Ward JL, Hirschmann PN (1979) In-vivo incorporation of $\left[{ }^{3} \mathrm{H}\right]$-proline into rabbit dentinal pulp collagen. Arch Oral Biol 24:613-615

Slavkin HC, Honig LS, Bringas P (1982) Experimental dissection of avian and murine tissue interactions using organ culture in a serumless medium free from exogenous (nondefined) factors. In: Sarnat B, Dixon AD (eds) Progress in Clinical and Biological Research (Vol 101), Factors and Mechanisms Influencing Bone Growth Alan R Liss, New York, pp 217-228

Weatherred JG (1965) Peripheral nervous system effects in the dental pulp of the rat and dog. Thesis. University of Texas, Austin

Weinstock M, Leblond CP (1974) Synthesis, migration, and release of precursor collagen by odontoblasts as visualized by radioautography after $\left[{ }^{3} \mathrm{H}\right]$-proline administration. J Cell Biol $60: 92-127$

Wright G, Leblond CP (1980) An attempt at quantitation of procollagens I and III and of collagen IV in tooth sections by exposure to the corresponding antibodies followed by ${ }^{125} \mathrm{I}$-protein $A$ and radioautography. $J$ Histochem Cytochem $28: 1355-1362$

Yamamura T (1985) Differentiation of pulpal cells and inductive influences of various matrices with reference to pulpal wound healing. J Dent Res 64:530-540

Yamamura T, Shimono M, Koike H, Terao M, Tanaka Y, Sakai Y, Inoue T, Yoshiki S, Tachikawa T, Kawahara H, Watanabe $\mathrm{O}(1980)$ Differentiation and induction of undifferentiated mesenchymal cells in tooth and periodontal tissue during wound healing and regeneration. Bull Tokyo Med Coll $21: 181-222$

Accepted August 28, 1986 